\title{
Healthcare and Teaching Hospitals
}

In order to improve healthcare for Brazilians, the Brazilian Health System (SUS) should guarantee a wide range of services, which include healthcare promotion, prevention, treatment and rehabilitation actions carried out in a long productive chain, from the small family healthcare unit to the large specialized hospital, which means from vaccination to organ transplantation, from analgesic to expensive surgical prostheses.

Each SUS service has its own characteristics, with specific techniques, ranging from behavioural approaches to the intensive use of equipment, with different degrees of complexity. They need trained professionals, technically and administratively, whose learning places are varied, with emphasis on teaching hospitals (TH).

The changes in the SUS thus include TH due to their participation in the training in the 14 professional healthcare categories and also because they constitute the most costly and complex SUS services'.

The healthcare sector is subject to the effects of demographic, epidemiological, technological, sociocultural, geopolitical and even climatic transitions, resulting in new and more costly demands on the system. These conditions increase the importance (and cost) of TH in researching and teaching new skills and medium and long-term strategies that make SUS sustainable ${ }^{2}$.

TH are classified in university hospital, hospital school and auxiliary teaching hospital. The first is owned or managed by universities and the second one by the medical school, while the third one is arranged with a higher education institution.

They are exponential organizations due to the constant and intense disruption and innovation of the technology used. It has high fixed costs for the capital invested in specific physical area, equipment whose technology is rapidly renewed, professionals of notorious specialization, expenses with research and education, high consumption of inputs, public utility and maintenance items, longer length of stay in hospital and minor turnover of beds.

There are in the country (connected to SUS) 200 TH certified, of which 51 are in the State of São Paulo (ESP), 11 belonging to the State Government.

In 2017, the $51 \mathrm{TH}$ admitted 710,000 patients (28.5\% of hospitalizations in the 614 hospitalization units of SUS/SP). The average of procedures of high complexity in SUS/SP is $8.4 \%$; in TH, it is $18.4 \%$. The mean value of hospitalization for TH is twice as high 
as the hospitalization value of other hospitals in the SUS/SP (according to the SUS table, which has very outdated values).

The monitoring of these units, both qualitative and quantitative, is carried out with information from both DATASUS and the Teaching Hospitals Evaluation System (SAHE) ${ }^{3}$.

The triad care, research and education do not include individualized assessments of quality, productivity and costs, new ways of measuring the operational outcome, which would allow better management and financing. Evaluation of qualitative and quantitative, institutional and professional performance, with publicity and transparency, give returns to society on the feasibility of programs and services mentioned.

The disorderly search of the population for TH services, recognized for their quality, as well as deficiencies in the SUS network generate operational problems. Cases of low complexity that could be better cared in less costly units, overcrowd their emergencies and ambulatories. The use of their beds as the back-end system for chronic and elderly patients reduce the access of more complex cases. Citizens covered by Supplementary Health (SS) use the TH services without the corresponding compensation.

Archaic models of administration also collaborate for poor indicators of turnover and hospital stay, for the lack of standardization of conducts and the use of materials and inputs, which make services more expensive.

The definition of lines of research based on healthcare policies will enable the integration between the objectives of the SUS, educational institutions, researchers and $\mathrm{TH}$, leading to the rationalization of the use of research resources. These resources should be clearly separated from the care expenses, (which is still a precarious fact), which would allow the good administrative performance of the unit.

Direct support from the government and through the development agencies (CAPES, FINEP, CNPq and FAPESP) and other entities has been fundamental for the advancement in this area. However, in the State of São Paulo, in 2017, 17 of the 51 TH have requested funds from FAPESP, which is lower than desired, but this has been increasing since 2006 when it began to be evaluated.
In the training of healthcare professionals, many measures need to be taken, in particular in TH. SUS managers, providers, professional trainers need to dialogue to decide on the profile of the healthcare professional that SUS needs for care and management ${ }^{4}$. The multi-professional participation of experts from the exact, social, biological and human sciences, makes possible the answer to the challenges. It is necessary to make a decision on load of knowledge, years of study and retraining for the type of action, practice and academic.

The offer of vacancies in specialization for trainee doctors, should be based on predictive scenarios, such as in countries that have studied trends from two to three decades onwards, defining residence and research funding bases. While institutions and TH are not aligned with the SUS and SS, hardly the new epidemiological and social challenges will be overcome. The state provides 6,600 medical residency grants for 53 programs, investing 270 million reais annually.

The use of public hospitals by private colleges is a practice that has been important, especially in ESP (with 59 medical schools), sometimes with more than one college using the same TH, a common fact in Brazil. It is essential to have an adequate 'student per bed' relation, giving respect and safety to the patient, learning opportunities, ethical and humanitarian aspects. Specific budgeting is imperative.

TH must have new models and administrative experiences, with flexible organizational structures, capable of promoting governability and sustainability, adaptive to the dynamics of the transitions and integration to SUS and SS. International experiences, partnerships and information exchange are necessary.

\section{REFERENCES}

1. Nogueira, D L, Lira, G V, Izabelle Albuquerque, I M A N, Linhares M S C. Avaliação dos Hospitais de Ensino no Brasil: uma Revisão Sistemática. Revista Brasileira de Educação Médica, 39 (1): 151-158; 2015

2. Mendes, J D V, Bittar, O | N V. Perspectivas e desafios na gestão pública do SUS, Rev. Fac. Ciênc. Méd. Sorocaba, v. 16, n. 1, p. 35 - 392014.

3. Bittar, O J N V, Magalhães A, Gouveia, R C A, Mendes, J D V. Saúde e protocolos de qualidade. Bol. Epidemiológico Paulista, BEPA Janeiro 2016; 13 (145): 19-32

4. Oliveira, MPR, Menezes I H C F, Sousa, L M, Peixoto, M R G. Formação e Qualificação de Profissionais de Saúde: Fatores Associados à Qualidade da Atenção Primária. Maria. Revista Brasileira de Educação Médica, 54740 (4): 547-559; 2016 